\title{
Aspectos Prácticos del Problema de Identificación de la Dinámica de Robots Industriales
}

\author{
Practical Aspects of the Dynamic Model Identification of Industrial Robots \\ Pablo S. Gonzalez \\ Facultad de Ingeniería, Universidad de Buenos Aires \\ Paseo Colón 850 (1063) Buenos Aires, Argentina \\ pgonzalefi.uba.ar \\ Recibido: 01/11/21; Aceptado: 01/12/21
}

\begin{abstract}
The practical aspects of the dynamical parameter identification method of an ABB IRB140 robot (off-line calibration procedure) are presented, such as identifiability, reduction to base parameters, inclusion of the measurements noise model and their influence on estimation uncertainty, scaling, and prior knowledge incorporation. A remarkable result is the calculation of the trajectories that provide a set of persistently excitatory signals of the dynamics in order to improve the estimation. The signals preprocessing is another relevant aspect since, to reduce the effect of noise, the excitation is limited in bandwidth and the derivatives of the joint positions are calculated in the transformed space.
\end{abstract}

Keywords: robot; parametric identification; robot dynamics trajectory optimization.

Resumen- Se presentan los aspectos prácticos del método de identificación de los parámetros dinámicos de un robot ABB IRB140 (procedimiento de calibración off-line), tales como la identificabilidad, la reducción a parámetros base, la inclusión del modelo del ruido en las mediciones y su influencia en la incertidumbre de la estimación, el escalamiento, y la incorporación del conocimiento previo. Un resultado destacable es el cálculo de las trayectorias que brindan un conjunto de señales persistentemente excitatorias de las dinámicas para mejorar la estimación, pasando la planificación del ensayo a ser una variable más del proceso de identificación. El tratamiento de las señales es otro aspecto relevante pues para reducir el efecto del ruido se limita en frecuencia a la excitación y las derivadas de las posiciones articulares se calculan en el espacio transformado.

Palabras clave: robot; identificación paramétrica; dinámica; optimización de trayectorias.

\section{INTRODUCCIÓN}

La dinámica de los robots articulados, es decir aquellos que tienen juntas de revolución que articulan los eslabones, presenta un comportamiento fuertemente no lineal. Los controladores más simples (por ejemplo PID) permiten estabilizar la planta y dar una respuesta satisfactoria en cuanto las prestaciones requeridas sean exigentes.

Para mejorar la performance es necesario considerar el comportamiento no lineal y el acoplamiento en la dinámica de los ejes. Por lo tanto una estrategia basada en $\mathrm{N}$ controladores SISO, tantos como ejes se tengan, no es suficiente. La técnica de control por torque computado [1], permite desacoplar y linealizar en forma exacta el modelo de la planta de manera que puede ser expresado por la siguiente ecuación,

$$
\mathbf{v}=\ddot{\mathbf{q}}
$$

donde la entrada es $\mathbf{v}$ y $\ddot{\mathbf{q}}$ es el vector de aceleraciones articulares. Luego las salidas del sistema son las posiciones de los ejes q y las velocidades articulares $\dot{\mathbf{q}}$.

Entonces el problema de control se reduce a reproducir una trayectoria de referencia en una planta compuesta de $\mathrm{N}$ doble integradores, para lo cual las técnicas de control lineal dan sobradas alternativas de elevado desempeño.

Este escenario se consigue siempre que la cancelación de las no-linealidades sea exacta, lo que implica contar con un modelo de la dinámica que describa de forma exacta las relaciones entre las variables.

El planteo del modelo de la dinámica expresa estas relaciones en función de una serie de parámetros, tales como las masas de los eslabones o los momentos y productos de inercia entre otros. La estructura del modelo obedece a los efectos considerados; por ejemplo, un modelo que contemple las elasticidades de las juntas tendrá una estructura diferente a aquel que no las incluye. Por lo tanto para tener un modelo exacto ${ }^{1}$ se necesita no solo incluir todos los efectos sino también establecer los valores de los parámetros para tal fin.

Es claro que no es posible acceder a un conocimiento absoluto y lo mejor que se puede hacer es acercarse en la menor medida posible, hecho que podríamos traducir en el conocimiento con menor incertidumbre de los parámetros dada una relación estructural que incluya los efectos preponderantes.

El control robusto y el control adaptativo proponen dos caminos para lidiar con este problema. En el primero de los casos se plantea un control que estabilice la familia de modelos descriptos por el conjunto de valores que pueden tomar los parámetros, dada la incertidumbre. En el segundo de los casos se busca identificarlos durante el movimiento adecuando el comportamiento del controlador.

La incertidumbre en los parámetros no solamente se explica en los términos tratados. También ocurre en situaciones tales como cuando el robot manipula un objeto desconocido; como el robot es una máquina que puede realizar tareas que no estén completamente determinadas a-priori, es natural encontrarse en esta situación. En este caso es importante partir de un valor inicial de los parámetros dinámicos para ajustar la estimación durante la ejecución de la tarea. Así se contempla una estrategia

\footnotetext{
${ }^{1}$ El sentido de esta expresión es referirnos a una construcción abstracta que intenta describir el comportamiento de un sistema de la manera más
} fiel posible. Tener presente la diferencia entre modelo y realidad 
off-line de caracterización de parámetros propios del brazo complementado con un algoritmo on-line que identifique la carga durante el movimiento.

El marco teórico en temas de identificación está nutrido por el trabajo de muchos grupos de investigación. En particular, en el Laboratorio de Robótica de FIUBA se cuentan como antecedentes dos tesis de grado correspondientes a Diego Zeida [2] y Alejandro Oubiña [3], la tesis de doctorado de Mauricio Anigstein [4], y varios artículos con referato. Los primeros trabajos publicados en el tema datan de mediados de la década de 1980. Hasta el día de la fecha se encuentra como un área activa, donde el interés reciente se enfoca en la mejora de los métodos de estimación y el tratamiento de las señales medidas.

\section{A. Organización del artículo.}

En la sección II se repasa el método de modelización de la dinámica, que establece las relaciones entre variables y parámetros. Luego en III se presenta el algoritmo de identificación, el cálculo del sesgo y la incertidumbre de la estimación. También se evalúan distintas técnicas para mejorar los resultados consistentes en el escalamiento, la reducción del modelo y la incorporación de conocimiento previo. En la sección IV-B se aborda el cálculo de la trayectoria óptima. Los resultados se presentan en VI seguidamente de las conclusiones.

\section{MODELO DE LA DINÁMICA DEL ROBOT}

Uno de los métodos para estudiar la dinámica de los manipuladores es mediante la resolución de la ecuación de Lagrange (ec. 1), donde el Lagrangiano $L$ se calcula como la resta entre la energía cinética $T(\mathbf{q}, \dot{\mathbf{q}})$ y la potencial $U(\mathbf{q})$, siendo las variables $\tau_{s}^{e}$ y $q_{s}$ las fuerza y posición generalizada sobre el eje $s$.

$$
\tau_{s}^{e}=\frac{d}{d t} \frac{\partial L}{\partial \dot{q}_{s}}-\frac{\partial L}{\partial q_{s}}
$$

El método de Lagrange permite obtener analíticamente un modelo en forma cerrada donde el principal desafío consiste en calcular $T$ y $U$.

La energía cinética puede expresarse entonces como una forma cuadrática según la ec. 2 .

$$
T=\frac{1}{2} \dot{\mathbf{q}}^{t} M \dot{\mathbf{q}}
$$

La matriz de inercia o de energía cinética $M$ se calcula según la ec. 3 , donde $A_{0}^{i}$ es la matriz homogénea de rototraslación que relaciona la terna asociada al eslabón $i$ con la terna inercial ó 0, y $J_{i}$ es la matriz de pseudoinercia del eslabón $i$ que incluye como parámetros a los momentos de inercia $\left(I_{o x x i}, I_{o y y i}, I_{o z z i}\right)$, los productos de inercia $\left(I_{o x y i}, I_{o x z i}, I_{o y z i}\right)$, la posición del centro de masas $\left(\mathbf{r}_{g}^{i}=\right.$ $\left.\left[\begin{array}{lll}x_{G i} & y_{G i} & z_{G i}\end{array}\right]^{t}\right)$ y su masa $\left(m_{i}\right)$, resultando en un total de 10 .

$$
m_{s j}=\sum_{i=\max (s, j)}^{N} \operatorname{Tr}\left[\frac{\partial A_{0}^{i}}{\partial q_{s}} J_{i} \frac{\partial A_{0}^{i}}{\partial q_{j}}\right]
$$

La energía potencial, desestimando el efecto elástico de articulaciones y considerando a los eslabones como cuerpos rígidos, se expresa según la ec. 4 , donde g es la aceleración de la gravedad y $\mathbf{r}_{g}^{i}$ es la posición del centro de masas del eslabón $i$ respecto de su terna.

$$
U=m_{i} \mathbf{g}^{t} A_{0}^{i} \mathbf{r}_{g}^{i}
$$

Reemplazando las ecs. 2 y 4 en 1 se obtiene en forma general el IDIM, o modelo de la dinámica inversa ${ }^{2}$ del manipulador, según la ec. 5 , donde se indica la dependencia de cada uno de los elementos $M, C$ y $\mathbf{G}$ que resultan mayormente en funciones no lineales de las posiciones y velocidades generalizadas.

$$
\boldsymbol{\tau}^{\mathbf{e}}=M(\mathbf{q}) \ddot{\mathbf{q}}+C(\mathbf{q}, \dot{\mathbf{q}}) \dot{\mathbf{q}}+\mathbf{G}(\mathbf{q})
$$

La matriz $C$ se obtiene derivando los componentes de $M$ según se expresa en la ec. 6 y los elementos del vector de torques producidos por peso propio $\mathbf{G}$ se calculan con la ec. 7 .

$$
\begin{gathered}
c_{s j}=\sum_{k=1}^{N} \frac{1}{2}\left(\frac{\partial m_{s j}}{\partial q_{k}}+\frac{\partial m_{s k}}{\partial q_{j}}-\frac{\partial m_{j k}}{\partial q_{s}}\right) \dot{q}_{k} \\
g_{s}=-\sum_{i=s}^{N} m_{i} \mathbf{g}^{T} \frac{\partial A_{0}^{i}}{\partial q_{s}} \mathbf{r}_{g}^{i}
\end{gathered}
$$

Luego al considerar los motores, las transmisiones y los reductores, se suman los momentos de inercia de los rotores, de los engranajes, y sus masas. Además por el hecho de que la unidad mecánica debe transportar los motores, la masa y la ubicación de los estatores y de los reductores incide sobre los parámetros dinámicos de los eslabones. Finalmente la pérdida producida en los rodamientos y en los componentes del reductor pueden modelizarse como rozamiento seco y viscoso. Todos estos efectos se deberían incorporar al planteo, previo a la resolución de la ec. 1 . Sin embargo, considerando que los ejes están actuados a través de reducciones de orden $N_{r} \sim 100$ se puede desestimar el débil acoplamiento dinámico entre el rotor del motor y el eslabón ${ }^{3}$, simplificando el planteo al resolver la ec. de Lagrange para el mecanismo del brazo sumando luego el efecto de los motores y las reducciones [5] . Se considera además que cada motor actúa solo sobre un eje, hecho que produce que la matriz $N_{r}$ resulte diagonal.

El modelo dinámico del actuador puede expresarse entonces según la ec. 8, donde $\tau$ es el vector de torques motor y los nuevos parámetros que se agregan son el momento de inercia equivalente $\left(J_{m}\right)$, el coeficiente de rozamiento viscoso $\left(B_{m}\right)$ y el de rozamiento seco o de Coulomb $\left(F_{c}\right)$.

$$
N_{r} \boldsymbol{\tau}=J_{m} N_{r}^{2} \ddot{\mathbf{q}}+B_{m} N_{r}^{2} \dot{\mathbf{q}}+F_{c} \tanh (\dot{\mathbf{q}})+\boldsymbol{\tau}^{e}
$$

Así las ecs. 5 y 8 definen el modelo de la dinámica del brazo con las simplificaciones propuestas. Ambas pueden

\footnotetext{
${ }^{2}$ El modelo directo permite implementar el simulador, y se obtiene fácilmente pues $M$ siempre tiene inversa

${ }^{3}$ Esta simplificación es equivalente a considerar que la velocidad angular del rotor del motor se debe únicamente a su propio giro, pues el efecto producido sobre él por el movimiento del mecanismo es al menos 2 ordenes de magnitud menor
} 
resumirse en la ec. 9, donde el conjunto de parámetros tiene un cardinal de 13 por eje.

$$
\boldsymbol{\tau}=\mathcal{M}(\mathbf{q}) \ddot{\mathbf{q}}+\mathbf{h}(\mathbf{q}, \dot{\mathbf{q}})
$$

La ec. 9 vincula los torques activos en los ejes con las variables de estado $\mathbf{x}^{t}=\left[\begin{array}{ll}\mathbf{q}^{t} & \dot{\mathbf{q}}^{t}\end{array}\right]$ y sus derivadas. Observando a continuación las ecs. 3, 6, 7 y 8, se concluye que las operaciones sobre los parámetros dinámicos son todas lineales si se considera las posiciones de los centros de masa multiplicadas por la masa $\left(m_{i} \mathbf{r}_{g}^{i}\right)$. Entonces el IDIM se puede reescribir según la ec. 10, donde la matriz $\phi$ es conocida como el regresor de la dinámica y $\mathbf{p}$ almacena los $N \times 13$ parámetros enunciados.

$$
\boldsymbol{\tau}=\phi(\mathbf{q}, \dot{\mathbf{q}}, \ddot{\mathbf{q}}) \mathbf{p}
$$

Como el método de cálculo presentado es general y sistematizado, se implementó un algoritmo que genera en forma automática el modelo dinámico para simular en Matlab y la función que implementa el regresor, a partir de los parámetros cinemáticos y dinámicos expresados en forma simbólica.

\section{IDENTIFICACIÓN PARAMÉTRICA POR MÍNIMOS CUADRADOS}

La identificación del IDIM puede tener como objetivo hallar un modelo estructural obteniendo así los parámetros físicos con la menor incertidumbre posible, o bien conseguir un modelo para la predicción donde el foco está puesto en el error de la salida. La identificación se plantea en este trabajo como un problema de ajuste o estimación de parámetros donde la meta es la minimización del error de predicción.

Una técnica habitual consiste en la aplicación de cuadrados mínimos. El planteo en este caso lleva a minimizar el error de predicción del IDIM según un ensayo donde se adquieren $K$ muestras de los torques aplicados $\boldsymbol{\tau}_{k}$ y sus posiciones y derivadas $\left(\mathbf{q}_{k}, \dot{\mathbf{q}}_{k}, \ddot{\mathbf{q}}_{k}\right)$. El regresor $\phi_{k}$ se calcula para cada una de las muestras y se plantea un problema lineal con $K N$ ecuaciones y $R$ incógnitas según la ec. 11, que puede expresarse en forma resumida en la ec. 12 , donde $\Phi$ es una matriz de $K N \times R$ elementos con los resultados apilados del regresor para las $K$ muestras, e es el vector de error y $\mathcal{T}$ es el vector de torques medidos.

$$
\begin{gathered}
{\left[\begin{array}{c}
\boldsymbol{\tau}_{1} \\
\vdots \\
\boldsymbol{\tau}_{K}
\end{array}\right]=\left[\begin{array}{c}
\phi_{1} \\
\vdots \\
\phi_{K}
\end{array}\right] \mathbf{p}} \\
\mathcal{T}=\Phi \mathbf{p}+\mathbf{e}
\end{gathered}
$$

La solución se obtiene, siempre que los $R$ valores singulares de $\Phi$ sean mayores que 0 , por medio de la pseudoinversa según la ec. 13.

$$
\hat{\mathbf{p}}=\left(\Phi^{t} \Phi\right)^{-1} \Phi^{t} \mathcal{T}
$$

El vector $\hat{\mathbf{p}}$ obtenido de esta manera minimiza $\|\mathbf{e}\|_{2}$.

En este punto surge investigar una serie de cuestiones sobre la posibilidad de realizar el cálculo de la ec. 13, y qué tan buenos son los resultados en caso de poder hallarse. Entonces se estudia la identificabilidad, la reducción a parámetros base, la incorporación de conocimiento previo, el efecto del ruido en la medición, y la adecuación de las muestras para mejorar el cálculo numérico.

\section{A. Identificabilidad y reducción del modelo}

En forma general no todos los parámetros pueden ser identificados debido a cuestiones referentes a la estructura de los manipuladores. Por ejemplo, en el robot IRB140 se observa que la masa del primer eslabón $\left(m_{1}\right)$ no puede ser hallada (aunque se puede obtener su momento de inercia sobre el eje de giro). Sin embargo si el manipulador se montara en un carro se podría excitar para observar $m_{1}$ aplicando fuerzas de desplazamiento en alguna dirección. Vale decir que a medida que uno avanza en la cadena cinemática más son los parámetros identificables pues las posibilidades de producir un movimiento más general son mayores cuánto más cerca se está de la herramienta. Esta limitación inicial se pone de manifiesto inclusive al observar la formulación del IDIM (ec. 9), pues de los $13 \times N$ parámetros que podrían estar presentes solo se observan $R$ de ellos.

Por otro lado, una deficiente selección de las muestras puede impedir la identificación de algún parámetro identificable. El regresor $\phi_{k}$ está compuesto de funciones que no forman una base ortogonal y por lo tanto se puede tener dependencia lineal entre ecuaciones. Sin llegar al caso extremo, si no se cuida que la selección de muestras produzcan que todas las funciones base sean expuestas se tiene un problema de pérdida de generalidad en la identificación que se manifiesta con menor performance del brazo al seguir otras trayectorias que las utilizadas para la identificación.

El número de condición de $\Phi$ indica cuan identificable es el sistema para un conjunto de muestras dadas. Habiendo sido lo suficientemente cuidadosos en la elección de ellas, la descomposición en valores singulares (ec. 14) muestra los parámetros que no pueden identificarse (aquellos asociados a valores singulares $s_{j}=0$ ) o los que de hacerlo se incurre en grandes errores $\left(s_{j}<\epsilon\right)$.

$$
\Phi=U \Sigma V^{t}
$$

Sin embargo, se debe apuntar que en este contexto los parámetros están en un espacio transformado por $V^{t}$ (ec. 15). Por lo que al detectar un valor singular muy pequeño no se descartaría un parámetro del modelo sino una combinación de ellos.

$$
\mathcal{T}=\sum_{j=1}^{r} s_{j}\left(\mathbf{v}_{j}^{t} \mathbf{p}\right) \mathbf{u}_{j}+\mathbf{e}_{j}
$$

La reducción del modelo consiste en todas las acciones tendientes a eliminar los parámetros que no pueden identificarse o son difícilmente identificables, y la discriminación procede en la observación de la matriz $\Sigma$. En [6] se propone un algoritmo donde para aquellos valores singulares $s_{j}$ tal que $s_{1} / s_{j}>100$ se inspecciona el vector columna $j$ de $V$, es decir $\mathbf{v}_{j}$, en busca de una componente dominante, que de hallarse indicaría un parámetro candidato a eliminar. Si se encontraran $r_{j}$ componentes dominantes, repitiéndose el patrón en $r_{j}$ ecuaciones entonces se está 
frente a una situación en la que los correspondientes parámetros pueden hallarse solo como combinación lineal, pudiendo plantearse una reducción a parámetros base mediante el método basado en la descomposición $Q R$ de la matriz de observación. Es claro que el nuevo conjunto reducido de parámetros $\mathbf{p}_{b}$ pierde el sentido físico. Los parámetros base también pueden obtenerse mediante la descomposición de $\Phi$ en valores singulares [7], [6] . En [3] y [2] se presenta un método iterativo para hallar las expresiones simbólicas de agrupamiento.

Una crítica al algoritmo de reducción del orden surge del hecho de trabajar con distintas escalas en los parámetros, siendo conveniente realizar un escalamiento previo (ver III-C) para no tomar decisiones erróneas. En todo caso siempre es mejor desestimar un parámetro cuando su influencia es pequeña que cuando es difícil de identificar.

La reducción de parámetros lleva a un modelo para la predicción, a menos que la información suprimida pueda suplirse de alguna forma, como por ejemplo incorporando el conocimiento previo conseguido por otros métodos.

Si se tienen $R-r$ parámetros que no pueden identificarse pero pueden ser determinados por otros métodos, partiendo de la ec. 10 se expresa la ec. 16, donde se han agrupado los términos conocidos con los supraíndices kn y los desconocidos con uk, se replantea el problema de estimación según la ec. 18.

$$
\begin{aligned}
\boldsymbol{\tau}_{k} & =\phi_{k}^{\mathrm{uk}} \mathbf{p}^{\mathrm{uk}}+\phi_{k}^{\mathrm{kn}} \mathbf{p}^{\mathrm{kn}}+\mathbf{e} \\
\boldsymbol{\tau}_{k}-\phi_{k}^{\mathrm{kn}} \mathbf{p}^{\mathrm{kn}} & =\phi_{k}^{\mathrm{uk}} \mathbf{p}^{\mathrm{uk}}+\mathbf{e} \\
\boldsymbol{\tau}_{k}^{\prime} & =\phi_{k}^{\mathrm{uk}} \mathbf{p}^{\mathrm{uk}}+\mathbf{e}
\end{aligned}
$$

\section{B. Modelo del ruido}

Durante el ensayo se relevan las posiciones $\mathbf{q}$ y sus derivadas $\dot{\mathbf{q}}$ y $\ddot{\mathbf{q}}$. Si se considera que la variable articular es tomada por un encoder de CPR pulsos por vuelta $(\Delta q=$ $2 \pi /$ CPR) y que el ruido no se debe a ningún otro factor más que a la discretización entonces se propone para el error de posición un modelo Gaussiano con media nula y desvío $\sigma_{p}=\Delta q /(2 \sqrt{3})$. Luego al aplicar un filtro derivador por diferencias sucesivas se tiene para la velocidad que el desvío es $\sigma_{v}=\sqrt{2} \sigma_{p} / T_{s}$, donde $T_{s}$ es el tiempo de muestreo y para la aceleración $\sigma_{a}=2 \sigma_{p} / T_{s}^{2}$. Siendo $T_{s} \ll 1$ es evidente la preponderancia del término $1 / T_{s}$ por lo que es indispensable acotar las componentes en frecuencia de las señales derivadas. Este primer grupo de incertidumbres impactan en el cálculo de $\Phi$.

En un segundo grupo se citan otras fuentes de error como las debidas a incertidumbres en las mediciones de los torques aplicados ( $\tilde{\mathbf{e}})$ caracterizadas por tener media nula y matriz de covarianza diagonal, y a dinámicas no modelizadas $\left(\mathbf{e}^{\mathrm{um}}\right)$, como rozamientos o comportamientos elásticos de ejes y eslabones, caracterizadas por variables aleatorias de media no nula y matriz de covarianza no diagonal. El error e en la ec. 12 es suma de ambas.

Si se considera solo el efecto del segundo grupo (es decir $\Phi$ determinístico), la esperanza del error de estimación $\tilde{\mathbf{p}}$ se calcula [8] según la ec. 19.

$$
E\{\tilde{\mathbf{p}}\}=-\left(\Phi^{t} \Phi\right)^{-1} \Phi^{t} E\{\mathbf{e}\}
$$

Por otro lado, considerando solo las del primer grupo y ruido de medición del torque, la covarianza del error de estimación resulta en la ec. 20, siendo su norma según la ec. 21 [9], donde se ha reemplazado el desvío estándar del ruido de medición.

$$
\begin{gathered}
\operatorname{cov}\{\tilde{\mathbf{p}}\}=E\left(\Phi^{t} \Phi\right)^{-1} E\left\{\mathbf{e e}^{t}\right\} \\
\|\operatorname{cov}\{\tilde{\mathbf{p}}\}\|=\sqrt{\frac{\sigma^{2}}{s_{\min }}}
\end{gathered}
$$

Si bien los resultados de las ecs. 19 y 20 son bien conocidos, son difícilmente aplicables en el caso que se presenta, ya que los efectos en ambos grupos se dan simultáneamente y el regresor es una función no lineal de las entradas por lo que se debilita la hipótesis de estacionariedad.

Sin embargo es esperable que la estimación tenga un sesgo (ec. 19), como así también que se pueda definir un intervalo de confianza a partir de la covarianza (ec. 20). En particular, esto último es especialmente útil porque permite acotar las estimaciones.

Partiendo de un conjunto de muestras e modelizada por un proceso de ruido blanco Gaussiano, la varianza muestral $s^{2}$ tiene una distribución $\chi^{2}$ con $\nu=K N-r$ grados de libertad y se puede definir un intervalo de confianza $\alpha$ (ec. 22) para la varianza.

$$
\sigma_{\min }^{2}=\frac{(K N-1) s^{2}}{\chi_{\frac{\alpha}{2}}^{2}}<\sigma^{2}<\frac{(K N-1) s^{2}}{\chi_{1-\frac{\alpha}{2}}^{2}}=\sigma_{\max }^{2}
$$

Por otro lado la estimación de la media tiene una distribución T-Student con $\nu$ grados de libertad, por lo que las cotas en las estimaciones de parámetros puede aproximarse para una determinada confianza $\alpha_{\text {par }}$ combinando los resultados de la ec. 22 para $\sigma_{\max }$ en 20 .

Finalmente, un punto destacable es el resultado [10] que muestra la forma en que se propagan los errores de medición (ec. 23) y de modelización hacia las estimaciones (ec. 24), dejando de manifiesto la importancia de realizar el ensayo con $\Phi$ lo mejor condicionada posible (cond $(\Phi)$ es el número de condición de la matriz de observación).

$$
\begin{aligned}
\frac{\|\tilde{\mathbf{p}}\|}{\|\hat{\mathbf{p}}\|} & \leq \operatorname{cond}(\Phi) \frac{\|\tilde{\mathcal{T}}\|}{\|\mathcal{T}\|} \\
\frac{\|\tilde{\mathbf{p}}\|}{\|\mathbf{p}\|} & \leq \operatorname{cond}(\Phi) \frac{\|\delta \Phi\|}{\|\Phi\|}
\end{aligned}
$$

\section{Escalamiento}

Los parámetros representan distintas magnitudes que cubren un rango amplio de valores, aumentando consecuentemente el número de condición de $\Phi$ sin significar esto una pobre selección de las muestras. Es decir que la resolución del problema numérico puede mejorarse definiendo un nuevo conjunto de valores que cubran la misma escala, aunque el efecto de propagación de los errores notado en las ecs. 23 y 24 quede inalterado por este artificio.

Adicionalmente se observa que si las fuerzas generalizadas tienen un rango muy disímil en cada 
eje también es conveniente realizar una transformación antes de la identificación. Esto lleva a una modificación de los parámetros identificados, hecho que no es significativo en un modelo para la predicción.

La propuesta habitual sobre el escalamiento de parámetros consiste en hallar el factor de escala como la inversa de la norma del correspondiente vector columna de $\Phi$. La ec. 12 se puede reescribir en la ec. 25 , donde se ha multiplicado y dividido por la norma del vector columna. Agrupando los términos y definiendo $h_{j}=1 /\left\|\boldsymbol{\Phi}_{j}\right\|$ se tiene la ec. 26 , donde $H=\operatorname{diag}(\mathbf{h})$.

$$
\begin{gathered}
\mathcal{T}=\sum_{j=1}^{r} \boldsymbol{\Phi}_{j} \frac{1}{\left\|\boldsymbol{\Phi}_{j}\right\|}\left\|\boldsymbol{\Phi}_{j}\right\| p_{j}+\mathbf{e} \\
\mathcal{T}=\Phi H H^{-1} \mathbf{p}+\mathbf{e}
\end{gathered}
$$

La nueva matriz de observación resulta $\Phi_{e}=\Phi H$ y tiene un menor número de condición.

\section{Incorporación de estimación previa}

Otro aspecto interesante es la posibilidad de incluir una estimación inicial de los parámetros, que puede ser obtenida por medición directa, extraídas de modelos $C A D$, o bien de otras experiencias previas. Esto es especialmente útil cuando se trabaja con algoritmos iterativos ya que aumenta la velocidad de convergencia. En cambio en el método offline podría servir para balancear los resultados pesando en mayor o menor medida la información previa con un método denominado como mínimos cuadrados amortiguados.

Contando con un valor inicial $\mathbf{p}_{0}$ se replantea la ec. 12 agregando el conocimiento inicial a continuación según la ec. 27, donde $I$ es la matriz identidad de dimensión dada por el espacio de parámetros.

$$
\left[\begin{array}{c}
\mathcal{T} \\
\mathbf{p}_{0}
\end{array}\right]=\left[\begin{array}{c}
\Phi \\
I
\end{array}\right] \mathbf{p}+\left[\begin{array}{l}
\mathbf{e} \\
\mathbf{0}
\end{array}\right]
$$

Definiendo $\breve{\mathbf{p}}=\mathbf{p}-\mathbf{p}_{0}, \breve{\mathcal{T}}=\mathcal{T}-\Phi \mathbf{p}_{0}$, e incorporando un parámetro $\lambda$ para regular el peso de $\mathbf{p}_{0}$ en la estimación final se tiene la ec. 28 , cuya solución por mínimos cuadrados resulta en la ec. 29 mostrando que el término $\lambda^{2} I$ ayuda al cálculo de la inversa. Expresándolo de otro modo, el aporte de la información puede suplir los problemas que se presentan cuando algún valor singular de $\Phi$ es muy bajo [6].

$$
\begin{gathered}
{\left[\begin{array}{c}
\breve{\mathcal{T}} \\
\mathbf{0}
\end{array}\right]=\left[\begin{array}{c}
\Phi \\
\lambda I
\end{array}\right] \breve{\mathbf{p}}+\left[\begin{array}{l}
\mathbf{e} \\
\mathbf{0}
\end{array}\right]} \\
\check{\mathbf{p}}=\left(\Phi^{t} \Phi+\lambda^{2} I\right)^{-1} \Phi^{t} \breve{\mathcal{T}}
\end{gathered}
$$

\section{PlanificACión DEL ENSAYO}

En III-B se exploró la importancia de contar con mediciones con la mayor relación señal a ruido posible, y la dificultad que presenta la determinación de las derivadas de la posición de los ejes en cuanto a esto.

En un esquema de identificación on-line la única alternativa es aplicar un filtro pasa bajos digital. La principal dificultad siempre presente es la decisión en cuanto a la frecuencia de corte, ya que como la trayectoria no puede fijarse de antemano entonces no se puede asumir nada respecto al ancho de banda de las señales. El criterio adoptado consiste en tomar la frecuencia de corte cinco veces por arriba de la frecuencia elástica [11] de manera de capturar en la mejor medida el comportamiento dinámico y atenuar el ruido de medición de frecuencias superiores. Una segunda limitante es que los filtros aplicados deben ser causales y por lo tanto inducen una distorsión de fase [12].

Por otro lado, en la identificación off-line se cuenta con la realización completa, permitiendo que las señales reciban otro tratamiento. En principio el ancho de banda de la excitación puede limitarse a una frecuencia conocida facilitando la derivación y el filtrado. Además las trayectorias de prueba se pueden repetir innumerables veces, y si el control del robot es lo suficientemente preciso el promedio de las realizaciones arroja una medición de la posición con sesgo nulo y varianza tan baja como sea posible.

\section{A. Trayectorias periódicas}

Considerando los beneficios de contar con una excitación periódica [13] [14], la trayectoria deseada se puede plantear (ec. 30) como suma de senoidales de una frecuencia base $\omega_{\text {per }}=2 \pi / T_{\text {per }}$, donde $T_{\text {per }}$ es el período.

$$
\mathbf{q}(t)=\mathbf{q}_{0}+\sum_{h=1}^{N_{h}}\left[\mathbf{A}_{h} \sin \left(h \omega_{\text {per }} t\right)+\mathbf{B}_{h} \cos \left(h \omega_{\text {per }} t\right)\right]
$$

El contenido armónico, y consecuentemente el ancho de banda, se limita con la variable $N_{h}$. Las derivadas temporales de la posición también resultan periódicas de ancho de banda acotado por $N_{h}$. Por otro lado la señal de torque también es periódica y su espectro limitado en frecuencia, pues el regresor $\phi$ incluye términos no lineales como multiplicación de velocidades y funciones senoidales de senoidales que a lo sumo duplican el contenido armónico.

Los parámetros de diseño son los valores medios $\mathbf{q}_{0} \mathrm{y}$ los vectores de amplitud $\mathbf{A}_{h}$ y $\mathbf{B}_{h}$ para cada armónica. La pulsación $\omega_{\text {per }}$ es preferentemente común a los ejes. En total se tienen $N\left(2 N_{h}+1\right)+1$ grados de libertad para especificar la trayectoria.

Para implementar esta técnica o bien se tiene acceso al controlador [14] y se llega con las referencias calculadas en la ec. 30 para $t=k T_{s}$, o se genera una trayectoria segmentada decimando el vector de muestras para usarlo como referencias en las intrucciones de movimientos joint cuidando que los errores de seguimiento sobre el trayecto deseado no sean significativos. Este aspecto es sumamente importante porque cualquier apartamiento implica un incremento del ancho de banda de las señales.

\section{B. Diseño óptimo de la trayectoria}

Una de las ventajas en cuanto a la señal de excitación en el esquema de identificación off-line es que forma parte del diseño del ensayo, por lo que puede ajustarse la trayectoria para excitar adecuadamente todas las características de la dinámica.

Un enfoque cuando se plantea un ensayo de identificación consiste en realizar movimientos simples en forma secuencial a la vez que se calculan las dinámicas que 
ellos excitan y se incorporan en el procedimiento como valores conocidos [15]. Si bien el método es sencillo y permite dirigir el análisis a unos pocos parámetros a la vez, se produce una acumulación de error. Así, resulta mejor realizar un movimiento sobre una trayectoria, tomando una cantidad de muestras sobre la misma que permita una minimización global del error [16]. Combinando ambas ideas, en [17] se propone un procedimiento basado en una serie de trayectorias que minimizan el número de condición de $\Phi$ y que excitan en forma separada los componentes de inercia, los acoplamientos inerciales, los términos de Coriolis, fuerza centrípeta y los de peso propio, donde la estimación de los parámetros se realiza al final considerando todos los datos colectados.

En la sección III-A se discutió la importancia de seleccionar adecuadamente las muestras del ensayo para poder excitar las dinámicas a identificar. Luego en III-B se mostró que la influencia de las perturbaciones sobre las estimaciones guardan relación a las muestras utilizadas para conformar el ensayo. Por lo tanto es conveniente preguntarse si se puede elegir el mejor conjunto de entrenamiento que maximice la identificabilidad y minimice la varianza de la estimación.

Se plantea así un problema de optimización, donde la función de costo se relaciona con los objetivos subrayados y las variables de ajuste son los parámetros que definen la trayectoria (ec. 30), que se encuentran restringidos a un dominio dado para que sean realizables por el robot. El problema de buscar la mejor trayectoria se traslada entonces a plantear la función de costo por un lado y expresar convenientemente las restricciones por otro.

Así se define primero un funcional, o función de costo $O$, cuyo extremo resuelve el problema. Una posibilidad es buscar el mínimo del número de condición de $\Phi$ [9] pues ambos objetivos se relacionan con él. De esta manera se busca que la excentricidad $s_{\max } / s_{\min }$ sea mínima y que la hiperelipsoide definida se asemeje a una hiperesfera. Otra opción equivalente es maximizar el menor de los valores singulares, resultando en la mejora del peor caso. En [10] y [16] se propone además, sumar en los funcionales anteriores términos relacionados a los máximos y mínimos de $\Phi$ para regular su escala. En [6] se reinterpreta el costo a través del alfabeto de optimalidades en relación con los criterios utilizados en robótica.

\section{Procesamiento de señales adquiridas}

Dada la naturaleza periódica de la señal de posición y torque, se puede considerar cada ciclo como la realización de un proceso estocástico. En cada instante de tiempo se tiene una variable aleatoria cuyos parámetros estadísticos se estiman como,

$$
\begin{aligned}
\bar{q}_{i, k} & =\frac{1}{N_{c}} \sum_{j=1}^{N_{c}} q_{i, k+j N_{s}} \\
\bar{\tau}_{i, k} & =\frac{1}{N_{c}} \sum_{j=1}^{N_{c}} \tau_{i, k+j N_{s}}
\end{aligned}
$$

$$
\begin{aligned}
\hat{\sigma}^{2}\left\{q_{i, k}\right\} & =\frac{1}{N_{c}-1} \sum_{j=1}^{N_{c}}\left(q_{i, k+j N_{s}}-\bar{q}_{i, k+j N_{s}}\right)^{2} \\
\hat{\sigma}^{2}\left\{\tau_{i, k}\right\} & =\frac{1}{N_{c}-1} \sum_{j=1}^{N_{c}}\left(\tau_{i, k+j N_{s}}-\bar{\tau}_{i, k+j N_{s}}\right)^{2}
\end{aligned}
$$

donde el índice $i$ indica el eje, $k$ es el número de muestra dentro del ciclo y va desde 1 hasta $N_{s}$ y $N_{c}$ es la cantidad de repeticiones.

A medida que la cantidad de ciclos aumenta es esperable que la varianza de la estimación de las señales promedio disminuya $\left(\sigma_{\bar{q}_{i, k}}^{2}, \sigma_{\bar{\tau}_{i, k}}^{2}\right)$, por lo que se puede considerar a las curvas de posición y torque promedios como punto de partida para la identificación.

Si se asume que las señales son suma de una función determinística, dadas por las curvas promedio, y un procesos estocástico estacionario de media nula, la varianza es constante y su estimación se encuentra promediando las halladas en las ecs. 31 y 32.

$$
\begin{aligned}
\hat{\sigma}_{q_{i}}^{2} & =\frac{1}{N_{s} N_{c}-1} \sum_{k=1}^{N_{s}} \sum_{j=1}^{N_{c}}\left(q_{i, k+j N_{s}}-\bar{q}_{i, k+j N_{s}}\right)^{2} \\
\hat{\sigma}_{\tau_{i}}^{2} & =\frac{1}{N_{s} N_{c}-1} \sum_{k=1}^{N_{s}} \sum_{j=1}^{N_{c}}\left(\tau_{i, k+j N_{s}}-\bar{\tau}_{i, k+j N_{s}}\right)^{2}
\end{aligned}
$$

El intervalo de confianza de las varianzas $\sigma_{q_{i}}^{2}$ y $\sigma_{\tau_{i}}^{2}$ se puede construir a partir de sus estimaciones según la ec. 22 .

Asumiendo que el controlador del robot es de muy alta performance, se suma como hipótesis que la dispersión en q se debe a ruido blanco Gaussiano aditivo, tal como se plantea en la sección III-B, y se puede limitar su influencia al mínimo con un filtro pasa bajos que aproveche el particular contenido en frecuencia propuesto para la trayectoria de excitación. Se calcula entonces el espectro utilizando la transformada discreta de Fourier y se elimina todo el contenido en frecuencias superiores a la pulsación $N_{h} \omega_{\text {per }}$.

Las derivadas de la posición articular se pueden calcular multiplicando su espectro filtrado $\mathbf{Q}(j \omega)$ por la variable compleja $j \omega$ y luego antitransformando para tener $\dot{\mathbf{q}}$, o por $-\omega^{2}$ y antitransformando para tener $\ddot{\mathbf{q}}$. Así se evita la aproximación de la derivada por restas sucesivas y la limitación que puede presentarse al no poder muestrear a altas frecuencias.

En cuanto a la señal de torque, el contenido armónico es mayor resultando en teoría como máximo el doble que el de la señal de posición. Se sigue entonces el mismo procedimiento duplicando la frecuencia de corte.

\section{VALIDACIÓN}

Si el objetivo de la identificación es obtener un modelo para la predicción, la validación puede realizarse comparando los resultados que arroja el modelo con las mediciones obtenidas para una tarea tipo. Por el contrario si el objetivo es determinar una estimación de los valores de los parámetros dinámicos la validación se realiza comparando con los valores convencionales verdaderos si son accesibles 


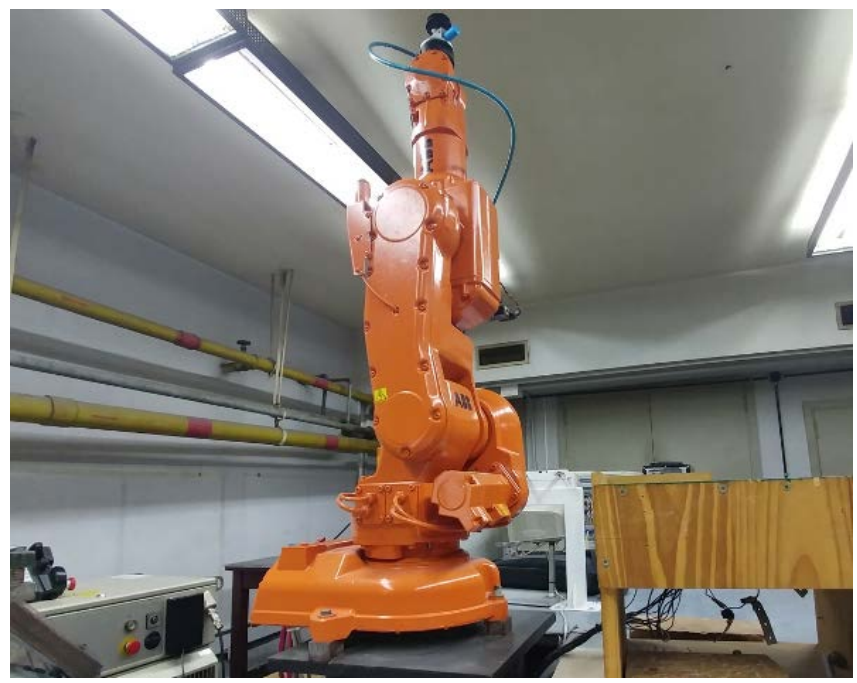

Fig. 1: Robot IRB140 del Laboratorio de Robótica de FIUBA

de algún modo ${ }^{4}$ o estimando las incertidumbres en la identificación y procurando que sean mínimas.

En [16] se recopilan otras alternativas frecuentemente adoptadas que se basan en comparar los resultados obtenidos para el mismo robot variando en un caso el conjunto de trayectorias de estimación, o la formulación del modelo en otro, como por ejemplo el modelo de potencia. Por otro lado se subraya que si el objetivo buscado es la mejora del control, una buena forma de validar la estimación es calificando el comportamiento del sistema en lazo cerrado.

En el presente trabajo la validación se realiza comparando medición contra predicción del modelo. En este punto es importante replicar el comportamiento de la planta considerando las tareas para las que fue pensada, aunque puede resultar engañoso ya que un robot en principio no está diseñado para resolver un problema en particular pues por definición es una máquina multipropósito. Por lo tanto, y para simplificar la evaluación, se consideran tareas básicas en las que tenga sentido definir la performance del movimiento [18], como por ejemplo desplazarse en línea recta entre dos puntos o describir una $U$ invertida tal como se realiza en una aplicación tipo pick and place. Es claro que cualquiera sea la tarea tipo propuesta para validar, ninguna de ellas tendrá las características impuestas a aquellas utilizadas durante la identificación.

La exigencia sobre la periodicidad puede ser mantenida repitiendo $N_{c}$ veces la tarea tipo y promediando las realizaciones para mejorar la relación señal a ruido de las mediciones de posición de los ejes y torques motor. Aunque no es posible tener un control fino sobre el ancho de banda por lo que se dificulta luego el filtrado.

\section{RESUltados}

Los temas teóricos y los aspectos prácticos presentados hasta aquí se aplican en la identificación del IDIM de un robot ABB-IRB140 (Fig. 1).

Para evaluar los aspectos de implementación se limita el estudio al brazo inferior, compuesto por los tres primeros

\footnotetext{
${ }^{4}$ Hecho sumamente improbable pues en tal caso no tendría demasiado sentido la tarea de identificación
}

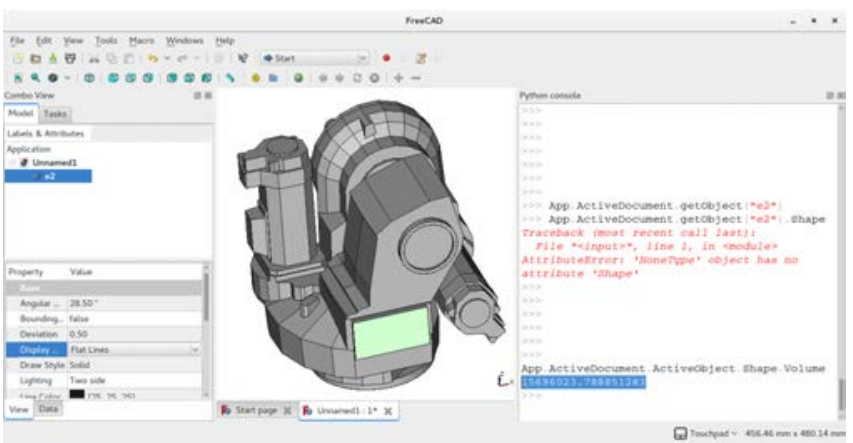

Fig. 2: Cálculo del volumen del eslabón 2 del robot IRB140 mediante FreeCAD

ejes, agrupando así los componentes articulados en la muñeca con el eslabón 3.

Al plantear las ecuaciones que definen el IDIM, se encuentra solo la influencia de 32 de $\operatorname{los} 13 \times 3=39$ parámetros iniciales. Luego se avanza en la reducción del conjunto de parámetros a estimar tomando del controlador del robot los momentos de inercia de los rotores de los motores $\left(J_{m}\right)$ y estimando los coeficientes de rozamiento viscoso $\left(B_{m}\right)$ especificados en motores de similares características.

En repetidas experiencias se observaron mejores resultados al sumar hipótesis sobre la masas de los eslabones. Se obtiene entonces el volumen de los eslabones 0, 1 (Fig. 2), 2, y agrupados el 3, 4, 5 y 6. Como en la hoja de datos figura que el peso de la unidad mecánica es $98 \mathrm{Kg}$, se calcula la densidad promedio y luego la masa de los grupos de eslabones en forma proporcional al volumen.

El regresor se separa en una parte conocida $\phi_{k n}$ (matriz de 9 columnas) y una desconocida $\phi_{u n}($ de $32-9=23$ columnas).

Los parámetros base se formulan armando la matriz de observación $\Phi$ a partir del regresor $\phi_{\text {un }}$ para 100 valores aleatorios de posición, velocidad y aceleración, aplicando luego el método discutido en la sección III-A. Así el conjunto de parámetros a identificar se reducen a 18 (ec. 33).

$$
\mathbf{p}_{b}=\left[\begin{array}{c}
I_{o y y 1}+I_{o y y 2}+I_{o y y 3}+0.14 m x_{G 1} \\
F_{c 1} \\
I_{o x x 2}-I_{o y y 2} \\
I_{o z z 2} \\
I_{o x y 2} \\
I_{o x z 2}-0.36 m z_{G 2}-0.36 m z_{G 3} \\
I_{o y z 2} \\
m x_{G 2} \\
m y_{G 2} \\
F_{c 2} \\
I_{o x x 3}-I_{o y y 3} \\
I_{o z z 3} \\
I_{o x y 3} \\
I_{o x z 3}-0.38 m z_{G 3} \\
I_{o y z 3} \\
m x_{G 3} \\
m y_{G 3} \\
F_{c 3}
\end{array}\right]
$$




\section{A. Trayectoria óptima}

Se propone una trayectoria de período $T_{p e r}=5 \mathrm{~s}$ compuesta por hasta 4 armónicos. El ancho de banda se limita entonces a $0.8 \mathrm{~Hz}$, permitiendo obtener en las señales de referencia del movimiento la suficiente riqueza como para excitar las dinámicas, siendo a la vez aceptable en cuanto a la frecuencia de muestreo de $20 \mathrm{~Hz}$ de un controlador de la serie 5 .

Los parámetros de amplitud y de valor medio de las señales (ec. 30) se calculan de manera de conseguir que la matriz de observación tenga un mínimo en el número de condición, acotando la excursión de la posición en un $80 \%$ de su rango y la de la velocidad y aceleración en un $70 \%$.

En la Fig. 3 se muestra la evolución de $\operatorname{cond}(\Phi)$ a la izquierda, y las señales que definen la trayectoria del ensayo a la derecha para cuando el proceso de optimización alcanzó la cota mínima del vector de incrementos.
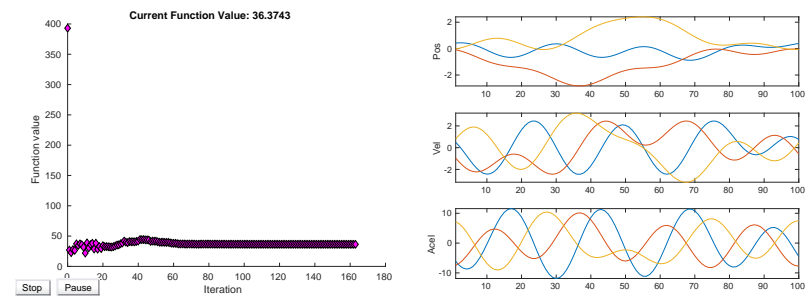

Fig. 3: Izq: evolución de $\operatorname{cond}(\Phi)$ a medida que se ajusta la trayectoria de prueba. Der: señales obtenidas

La trayectoria óptima hallada se puede reproducir con movimientos joint entre 20 destinos dados en coordenadas articulares mostrados en la tabla I y distanciados $250 \mathrm{~ms}$.

TABLE I: Coordenadas joint de la Trayectoria Optima

\begin{tabular}{|c|c|c|c|}
\hline $\begin{array}{r}\text { Tiempo } \\
{[\mathrm{s}]}\end{array}$ & $\begin{array}{r}q_{1} \\
\text { deg }\end{array}$ & $\begin{array}{r}q_{2} \\
\text { deg }\end{array}$ & $\begin{array}{r}\text { q3 } \\
\text { deg }\end{array}$ \\
\hline 0.25 & 24.098 & 84.657 & -181.197 \\
\hline 0.50 & 17.286 & 60.213 & -159.677 \\
\hline 0.75 & -13.739 & 30.426 & -137.475 \\
\hline 1.00 & -37.406 & 13.867 & -140.872 \\
\hline 1.25 & -25.228 & 4.204 & -166.747 \\
\hline 1.50 & 7.936 & -17.576 & -184.000 \\
\hline 1.75 & 19.932 & -51.261 & -168.327 \\
\hline 2.00 & -4.650 & -71.724 & -126.987 \\
\hline 2.25 & -34.243 & -59.435 & -85.765 \\
\hline 2.50 & -32.008 & -26.323 & -59.771 \\
\hline 2.75 & -4.401 & -1.225 & -46.267 \\
\hline 3.00 & 8.146 & 6.087 & -42.475 \\
\hline 3.25 & -14.428 & 14.048 & -56.444 \\
\hline 3.50 & -45.251 & 39.973 & -92.940 \\
\hline 3.75 & -46.080 & 73.231 & -136.702 \\
\hline 4.00 & -15.988 & 88.000 & -162.384 \\
\hline 4.25 & 11.171 & 77.988 & -162.589 \\
\hline 4.50 & 13.406 & 65.175 & -156.354 \\
\hline 4.75 & 5.658 & 70.291 & -163.972 \\
\hline 5.00 & 11.268 & 85.232 & -180.023 \\
\hline
\end{tabular}

\section{B. Identificación sobre el simulador de $A B B$}

El entorno de programación off-line que complementa los sistemas robóticos de ABB incluye un completo simulador cinemático y dinámico para reproducir fielmente las tareas de las aplicaciones robóticas antes de que ellas sean instaladas en planta. Por tratarse de software cerrado no se tiene acceso a los modelos ni los parámetros utilizados. La experiencia de trabajar con el software es similar en muchos aspectos a hacerlo con el robot real.

Al igual que los robotistas ensayan sus aplicaciones en el entorno de software, en el presente trabajo la identificación del IDIM se realiza en el ambiente virtual. Habiendo probado la reproducción de las trayectorias y teniendo un modelo identificado, se puede replicar la experiencia con el robot real utilizando los mismos programas. En nuestro caso surge adicionalmente una serie de dificultades [19] por tratarse de una versión de controlador anterior:

- Período de muestreo. el modelo del Laboratorio de Robótica limita las interrupciones cíclicas a $250 \mathrm{~ms}$, aunque permite realizar interrupciones no cíclicas a 50 ms. Esto impacta en la tasa de captura de datos.

- Acceso a la señal de torque. La versión SC4-M2000 del controlador permite leer el torque de referencia a través de la función TestSignRead, aunque limita su aplicación a ejes externos. Se necesita entonces medir externamente la corriente en los motores sincronizando la adquisición con el robot.

La trayectoria del ensayo se ejecuta en el entorno virtual (Fig. 4), y los datos adquiridos de posición y torque de los 3 primeros ejes se salvan en un archivo. El primer y último ciclo se descartan porque durante parte de ellos el robot acelera desde el reposo en un caso y en el otro frena hasta detenerse. En la figura se observa que los trazos de las distintas realizaciones son coincidentes, salvo el del ciclo inicial.

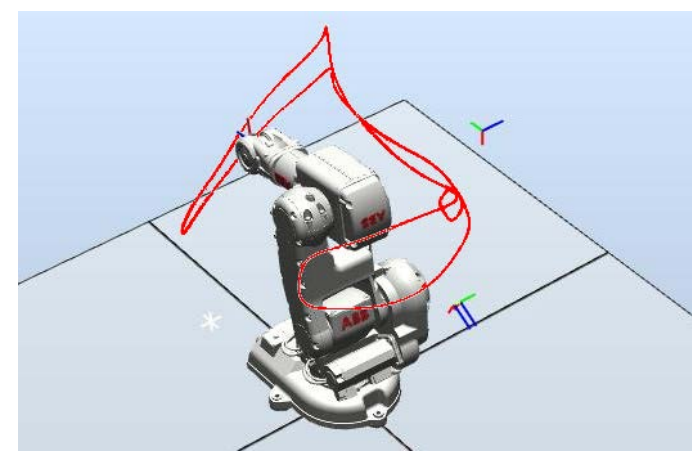

Fig. 4: Ejecución del ensayo en RobotStudio

1) Procesamiento de los datos adquiridos: El tiempo de la interrupción cíclica destinada a tomar las muestras tiene una variabilidad que llega a $2 \mathrm{~ms}$. Se puede modelizar como una dispersión de $0.5 \mathrm{~ms}$ al rededor de $51.5 \mathrm{~ms}$. Así los datos adquiridos son tomados en intervalos de muestreo variables, por lo que el primer paso consiste en regularizarlo. Como además se deben identificar con el menor error los instantes de inicio de cada ciclo para no tener realizaciones desfasadas que influyan negativamente en la estimación de los datos de posición y torque, se submuestrean los datos con $T_{s}=10$ ms. En la Fig. 5 se muestran los datos de posición y torque interpolados. 

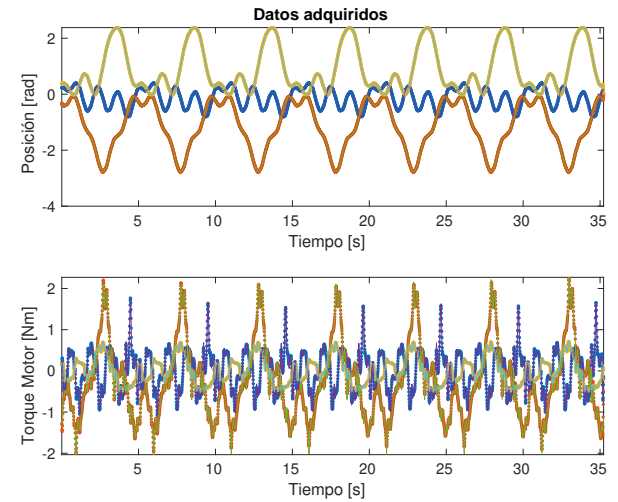

Fig. 5: Datos submuestreados con un período $T_{s}=10 \mathrm{~ms}$

El inicio de cada ciclo se obtiene buscando los máximos de la autocorrelación de la señal de posición en un entorno correspondiente al tiempo de ciclo propuesto para la señal del ensayo. En el caso presentado basándose en la trayectoria propuesta cada ciclo debiera tener 500 muestras espaciadas $10 \mathrm{~ms}$, pero luego de analizar los datos se encuentra que son en realidad 504 muestras por ciclo.

El espectro de las curvas de posición y torque promedios se muestran en la Fig. 6. La posición presenta un ancho de banda de $0.8 \mathrm{~Hz}$, aunque el espectro del torque resulta más disperso. Se proponen las frecuencias de corte $f_{c q}=2.4 \mathrm{~Hz}$ para la señal de posición y $f_{c u}=4.8 \mathrm{~Hz}$ para la del torque.
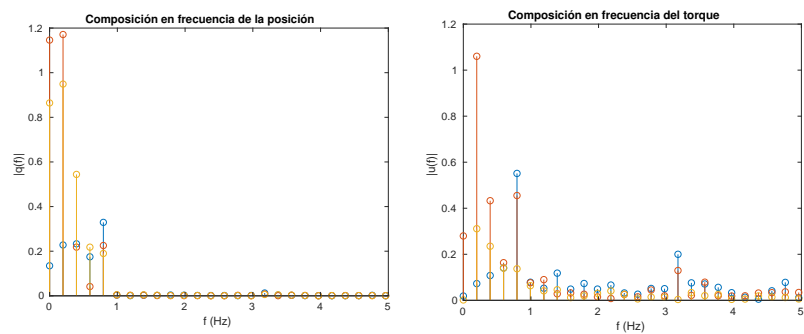

Fig. 6: Espectros de las señales promediadas de posición y torque

En la Fig. 7 se observa a derecha la estimación de la posición superpuesta a los datos de los ciclos muestreados y a la izquierda la curva de velocidad calculada en el dominio transformado.
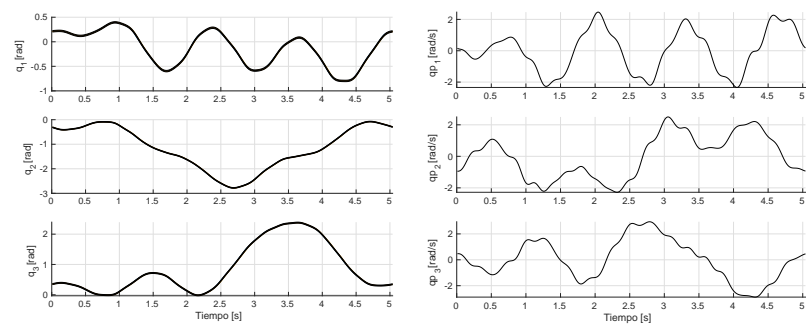

Fig. 7: Señales de posición y velocidad estimadas

En la Fig. 8 se presentan la aceleración y el torque motor estimados. Se superpone en la gráfica del torque las mediciones tomadas en todos los ciclos. La estimación del torque se grafica junto con las bandas de máximo y mínimo valor caracterizadas a partir de la varianza muestral de las mediciones para incluir el $95 \%$ de las muestras.
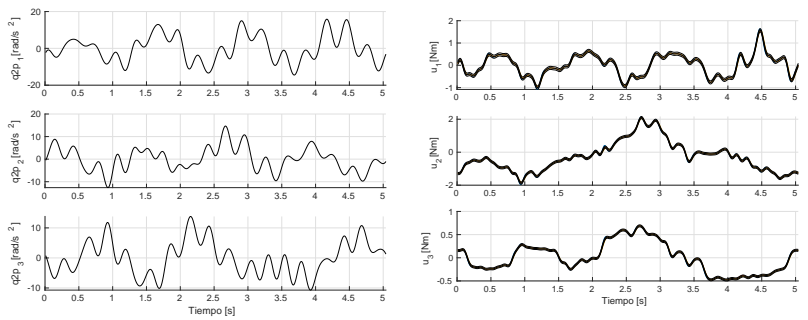

Fig. 8: Señales de aceleración y torque estimadas

2) Resultado de la identificación: Con los datos preprocesados se corre la identificación. La estimación lineal sirve de condición inicial de un proceso de identificación no lineal e iterativo basado en la estimación previa de la covarianza del torque. Así se refinan los parámetros dando mayor peso a las mediciones más confiables. En la tabla II se muestran los valores estimados de los parámetros base junto con su intervalo de confianza del $95 \%$.

TABLE II: Parámetros Base Estimados

\begin{tabular}{r|r|r|r|}
\hline Param & Ident & Min & Max \\
\hline$I_{o y y 1}+I_{o y y 2}+I_{o y y 3}+0.14 m x_{G 1}$ & 6.2672 & 6.2651 & 6.2693 \\
$F_{c 1}$ & 0.45214 & 0.45203 & 0.45225 \\
$I_{o x x 22}-I_{o y y 2}$ & -2.1315 & -2.1343 & -2.1287 \\
$I_{o z z 2}$ & 1.9237 & 1.9218 & 1.9256 \\
$I_{o x y 2}$ & 0.10048 & 0.098863 & 0.10209 \\
$I_{o x z 2}-0.36 m z_{G 2}-0.36 m z_{G 3}$ & 0.34712 & 0.3459 & 0.34834 \\
$I_{o y z 2}$ & 0.18018 & 0.17905 & 0.18131 \\
$m x_{G 2}$ & -8.1397 & -8.1408 & -8.1386 \\
$m y_{G 2}$ & -0.40546 & -0.40657 & -0.40434 \\
$F_{c 2}$ & 0.40672 & 0.40663 & 0.40681 \\
$I_{o x x 3}-I_{o y y 3}$ & -3.3542 & -3.3552 & -3.3532 \\
$I_{o z z 3}$ & 4.0412 & 4.0409 & 4.0414 \\
$I_{o x y 3}$ & -0.62208 & -0.62266 & -0.62151 \\
$I_{o x z 3}-0.38 m z_{G 3}$ & -0.29632 & -0.29656 & -0.29609 \\
$I_{o y z 3}$ & 0.17383 & 0.17367 & 0.17399 \\
$m x_{G 3}$ & -9.6518 & -9.6519 & -9.6516 \\
$m y_{G 3}$ & -2.2081 & -2.2083 & -2.2078 \\
$F_{c 3}$ & 0.28581 & 0.2858 & 0.28582 \\
\hline
\end{tabular}

Para evaluar la bondad del ajuste, se corre la validación sobre los datos de identificación (Fig. 9).
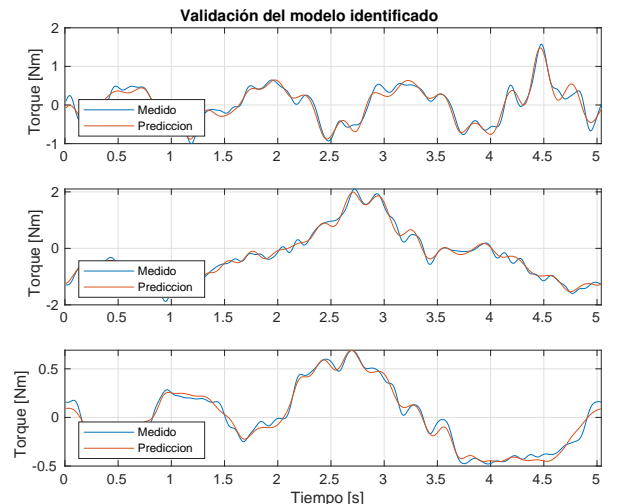

Fig. 9: Comparación del torque estimado para la tarea de identificación y su predicción por el modelo identificado para los 3 ejes

Se realiza una nueva identificación para evaluar la incidencia de la frecuencia de corte de los filtros aplicados al preprocesamiento, limitando el espectro de los datos hasta las frecuencias $f_{c q}=1.2 \mathrm{~Hz}$ y $f_{c u}=2.4 \mathrm{~Hz}$. Con esta nueva identificación se busca que el modelo refleje mejor las tendencias. En la Fig. 10 se observa que el ajuste resulta más grosero. 

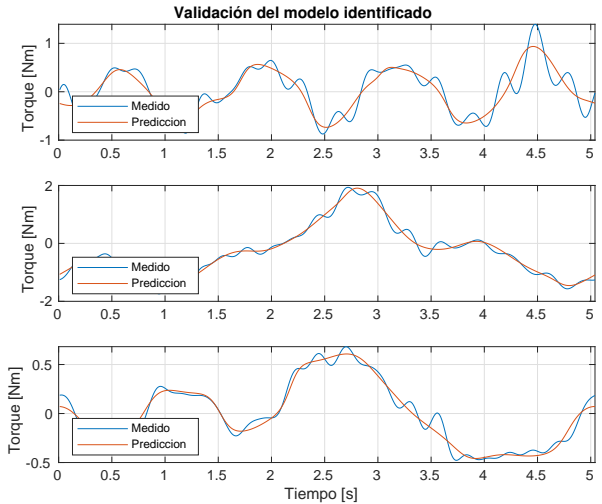

Fig. 10: Comparación del torque estimado para la tarea de identificación y su predicción considerando $f_{c q}=1.2 \mathrm{~Hz}$

\section{Validación}

El modelo identificado se valida evaluando su capacidad de predicción del torque necesario para realizar la tarea típica que se muestra en la Fig. 11.

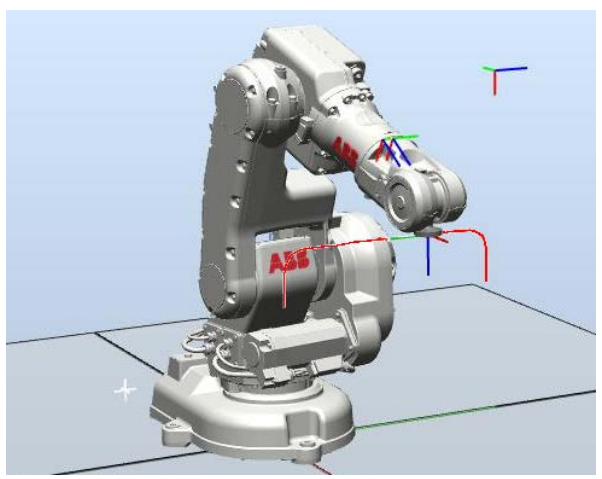

Fig. 11: Ejecución de la trayectoria de validación en RobotStudio

Las curvas capturadas en este caso están compuestas por menor cantidad de muestras. El procedimiento para procesarlas es el mismo al detallado para la trayectoria de identificación. En la Fig. 12 se muestran las señales estimadas y su intervalo de confianza del $95 \%$ comparadas con los datos preprocesados.
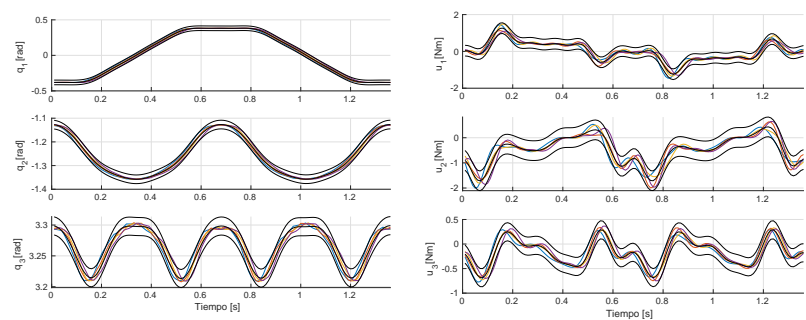

Fig. 12: Señales de posición y torque estimadas comparadas con los datos adquiridos

En la Fig. 13 se muestra la curva de torque estimada comparada con la predicción. En todos los ejes la predicción resulta dentro del intervalo de confianza de la estimación del torque. Se observa que la predicción aparece como una curva promedio de espectro acotado en relación a la estimada a partir de los datos.
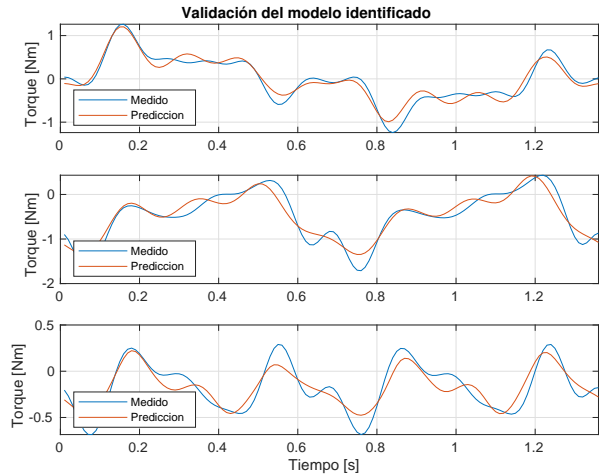

Fig. 13: Comparación del torque estimado y su predicción

Luego se evalúa la capacidad de predicción del modelo identificado usando filtros con frecuencias de corte $f_{c q}=$ $1.2 \mathrm{~Hz}$ y $f_{c u}=2.4 \mathrm{~Hz}$. En la Fig 14 se observa que si bien este modelo no ajusta adecuadamente las muestras de identificación, produce mejores resultados en cuanto a la predicción.
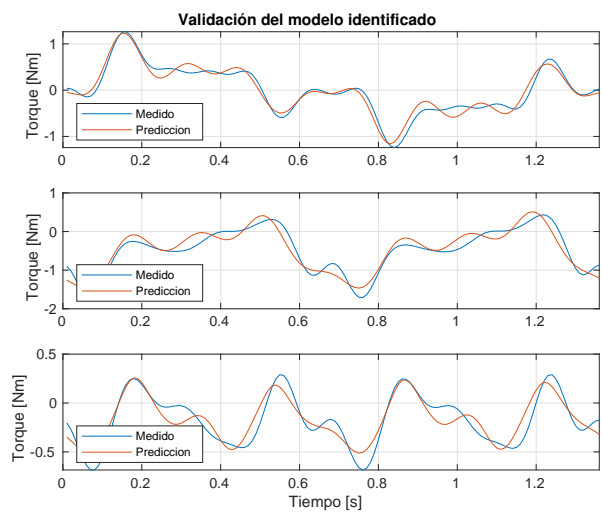

Fig. 14: Comparación del torque estimado y su predicción por el modelo identificado para los 3 ejes, considerando $f_{c q}=1.2 \mathrm{~Hz}$ en la identificación

\section{CONCLUSIONES}

Se presentó el marco teórico que permite derivar el modelo paramétrico de la dinámica de un manipulador serie en forma sistemática. Atendiendo a la imposibilidad de realizar mediciones directas de ciertos parámetros ya sea porque implique desensamblar un robot o bien porque ellos se manifiestan solo durante el movimiento, se planteó el problema de la identificación. Un primer enfoque se basó en el ajuste por mínimos cuadrados aprovechando la posibilidad de reformular el modelo dinámico inverso en una versión lineal en los parámetros. Para refinar la estimación se incluyeron luego algoritmos no-lineales iterativos. Como el objetivo del ajuste está puesto en minimizar el error cuadrático medio entre la estimación y el conjunto de datos medidos se produce un modelo con una buena capacidad de predicción, sustentando de esta forma el objetivo del trabajo que consiste en generar un modelo útil para el estudio del control, dejando en un segundo plano la idea de tener una buena medición de ciertos parámetros dinámicos.

Se detectó la influencia en el modelo de un conjunto de parámetros y se concluyó que correspondían al conjunto mínimo identificable o parámetros base. 
Se estudió el modelo del ruido y la incidencia en las estimaciones de los parámetros. Se presentó un marco estadístico que justifica la validez de las estimaciones y permite evaluar la calidad de las mismas.

Para mejorar el tratamiento de las señales, atendiendo a que el muestreo está limitado a una muy baja frecuencia, y que solo se cuenta con señales de posición teniendo que estimar las velocidades y aceleraciones de los ejes, se incorporó un esquema de trayectorias de identificación periódicas limitadas en ancho de banda. Así se redujo el ruido de medición de las señales de torque y posición a la vez que se implementó el cálculo de las velocidades y aceleraciones en el espacio transformado.

Se incorporó el diseño del ensayo al procedimiento de identificación, generando la trayectoria para maximizar la excitación de las dinámicas asociada a los parámetros por un lado, y adecuándolas a las posibilidades de reproducción del robot. Se comprobó que la capacidad de predicción del modelo identificado mejora en virtud de la selección de la trayectoria de identificación.

Finalmente se trabajó sobre el modelo IRB140 colectando los datos mediante el simulador del RobotStudio, e identificando luego su IDIM que puede ser aplicado en la investigación de técnicas de control entre otros.

Como trabajos futuros se destacan, la ejecución de las trayectorias de prueba en el robot IRB140 del laboratorio. En este caso aparece un desafío adicional debido a una limitación en relación a la adquisición de datos.

\section{REFERENCES}

[1] M. W. Spong and M. Vidyasagar, Robot Dynamics and Control. NY: Wiley \& Sons, 1989.

[2] D. Zeida, "Desarrollo de un paquete de simulación para robots en un ambiente con manejo de ecuaciones simbólicas (mathemática)." Tesina de Graduación en Ingeniería Electrónica, FIUBA, 1996.

[3] A. Oubiña, "Identificación de parámetros dinámicos de robots para calibración y control adaptativo.” Tesis de Graduación en Ingeniería Electrónica, FIUBA, 1996

[4] M. Anigstein, "Dinámica de manipuladores robóticos." Tesis Doctoral, FIUBA, 2002.

[5] A. Luca and W. Book, Robots with Flexible Elements, pp. 287-319. 012008.

[6] J. M. Hollerbach, W. Khalil, and M. Gautier, "Model identification," in Springer Handbook of Robotics, pp. 113-138, 2016.

[7] C. Atkeson, C. An, and J. Hollerbach, "Estimation of inertial parameters of manipulator loads and links," Int J Robot Res, vol. 5, no. 3, pp. 101-119, 1986.

[8] M. Gautier, "Identification of robots dynamics," IFAC Proceedings Volumes, vol. 19, no. 14, pp. 125-130, 1986.

[9] B. Armstrong, "On finding exciting trajectories for identification experiments involving systems with nonlinear dynamics," The International Journal of Robotics Research, vol. 8, no. 6, pp. 2848, 1989.

[10] M. Gautier and W. Khalil, "Exciting trajectories for the identification of base inertial parameters of robots," The International Journal of Robotics Research, vol. 11, no. 4, pp. 362-375, 1992.

[11] M. Brunot, A. Janot, P. Young, and F. Carrillo, "An improved instrumental variable method for industrial robot model identification," Control Engineering Practice, vol. 74, 032018.

[12] W. Khalil and E. Dombre, Modeling, Identification and Control of Robots. USA: Taylor \& Francis, Inc., 2002.

[13] J. Swevers, C. Ganseman, D. B. Tukel, J. de Schutter, and H. Van Brussel, "Optimal robot excitation and identification," IEEE Transactions on Robotics and Automation, vol. 13, pp. 730-740, Oct 1997.

[14] J. Swevers, W. Verdonck, and J. Schutter, "Dynamic model identification for industrial robots," Control Systems, IEEE, vol. 27, pp. $58-71,112007$.
[15] K. Yoshida, N. Ikeda, and H. Mayeda, "Experimental study of the identification methods for an industrial robot manipulator," in Proceedings of the IEEE/RSJ International Conference on Intelligent Robots and Systems, vol. 1, pp. 263-270, July 1992.

[16] J. Wu, J. Wang, and Z. You, "An overview of dynamic parameter identification of robots," Robotics and Computer-Integrated Manufacturing, vol. 26, no. 5, pp. 414-419, 2010.

[17] P. O. Vandanjon, M. Gautier, and P. Desbats, "Identification of robots inertial parameters by means of spectrum analysis," in Proceedings of 1995 IEEE International Conference on Robotics and Automation, vol. 3, pp. 3033-3038 vol.3, May 1995.

[18] ISO, "Manipulating industrial robots - performance criteria and related test methods," standard, International Organization for Standardization, Geneva, CH, Apr. 1998.

[19] ABB, System Data Types and Routines. ABB Robotics AB, S-721 68 Vasteras, Sweden, 4.0 ed., 2000. 